\title{
Multilinguales
}

\section{La syllabe et la découverte du sens dans l'énonciation : phonologie et langues en contact en FLE}

The Syllable and the Discovery of Meaning in the Statement: Phonology and Languages in Contact in FLE

\section{Maria-Luisa Fernandez-Echevarria}

\section{(2) OpenEdition Journals}

Édition électronique

URL : http://journals.openedition.org/multilinguales/2295

DOI : $10.4000 /$ multilinguales.2295

ISSN : 2335-1853

\section{Éditeur}

Université Abderrahmane Mira - Bejaia

\section{Édition imprimée}

Date de publication : 1 décembre 2013

Pagination : 43-60

ISSN : 2335-1535

\section{Référence électronique}

Maria-Luisa Fernandez-Echevarria, "La syllabe et la découverte du sens dans l'énonciation : phonologie et langues en contact en FLE », Multilinguales [En ligne], 2 | 2013, mis en ligne le 01 décembre 2013, consulté le 17 septembre 2019. URL : http://journals.openedition.org/multilinguales/ 2295 ; DOI : 10.4000/multilinguales.2295

Ce document a été généré automatiquement le 17 septembre 2019.

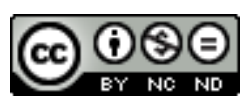

Multilinguales est mise à disposition selon les termes de la Licence Creative Commons Attribution Pas d'Utilisation Commerciale - Pas de Modification 4.0 International 


\section{La syllabe et la découverte du sens dans l'énonciation : phonologie et langues en contact en FLE}

The Syllable and the Discovery of Meaning in the Statement: Phonology and Languages in Contact in FLE

Maria-Luisa Fernandez-Echevarria

1 Pour construire un gabarit syllabique opératif dans l'acte de communication, l'émetteur et le récepteur du message doivent s'accorder sur des images acoustiques responsables de la transmission de l'information. Nous cherchons, dans quelques exemples de discours de la publicité et de la presse, l'origine des faits linguistiques qui rendent le message de la propagande effectif par la richesse des indices iconiques qu'il produit.

2 D'un point de vue strictement linguistique, les images à impact sont déclenchées par la souplesse accordée aux constituants syllabiques qui dynamisent des mots phonétiques figés au moment même de l'acte de communication. Les apprenants des langues étrangères sont bien plus souvent confrontés à la langue écrite qu'à l'interaction. Nous étudions alors, dans un corpus de FLE, la syntaxe qui a permis de transcrire des conversations et de considérer l'énonciation signifiante malgré le caractère fixe que l'on doit accorder aux constituants d'un texte transcrit.

3 Manquant de souplesse, la syllabation se comporte comme les expressions de la parémiologie dans le discours expert; celles-ci signifient dans le cadre macrosyntaxique et transmettent une information iconique qui ne repose pas exclusivement sur la typologie de ses composants.

\section{Français oral et code scriptural}

4 Le français, ayant perdu grand nombre de marqueurs morpho-phonologiques, présente, pour l'apprenant, un code d'écriture opaque souvent source de mauvaises 
interprétations syntaxiques (liaisons et enchaînements fautifs) en lecture et en conversation. Les phénomènes de déplacement de positions métriques (Encrevé, 1988 ; Laks, 1997, 2005) ne sont pas reconnus et produisent des erreurs dans l'expression du signifié. Par exemple, les syntagmes nominaux /le?оtœь/ (les hauteurs) et /lezotœb/ (les auteurs) n'ont évidemment pas un même référent, mais l'apprenant aura tendance à produire, par une mauvaise interprétation du code alphabétique, un même mot phonétique. On transcrira alors/leotœs/,/les'otœs/ ou/lezotœs/ indifféremment pour les deux interprétations alternatives, ce qui provoquera de mauvaises habitudes de segmentation.

5 L'énoncé n'est donc souvent pas construit par rapport à une macro-syntaxe informationnelle (Martin, 2009 ; Rossi, 1979) et la construction textuelle devient alors de plus en plus compliquée. Les erreurs d'interprétation phonétique du code écrit n'empêchent cependant pas toujours l'efficacité communicative, car l'interlocuteur dispose d'autres sources d'information (pragmatique, référentielle, gestuelle,...) qui ne sont pas strictement linguistiques.

6 Les erreurs réitératives ne sont donc pas surprenantes. Présenter la syllabation ou plutôt la constituance syllabique (Laks, 1997), une fois que l'image acoustique du mot est présente dans l'inter-langue du locuteur, pourrait être une remédiation à ce problème. On présenterait alors le déclencheur iconique avant que la verbalisation ne soit réalisée.

7 Une nouvelle approche des pratiques de classe, fondée sur le français oral et centrée sur les productions des apprenants peut être ainsi envisagée en prenant en considération les différents types d'énonciation. Cette approche n'est pas incompatible avec d'autres analyses linguistiques des genres discursifs.

\section{La syllabe phonologique}

8 La syllabe se modèle et se construit dans la dynamique du discours et non pas l'inverse. Comment justifier autrement l'expression créative? Quelles occurrences seront adoptées dans le répertoire de la langue? Comment pouvoir tout simplement s'exprimer si la syllabe est prise dans un moule indéformable?

9 Partant de la verbalisation d'un énoncé interprété, on peut reconstruire la structure syllabique qui n'est autre que l'intention du sujet parlant voulant communiquer par la syntaxe des éléments sonores. Aborder la syllabe, en partant du discours dans lequel elle s'inscrit, implique de décrire les phénomènes phonologiques (auto-segmentaux) qui ne sont pas directement accessibles à la syllabation que l'on en fait par écrit. Martin $\mathrm{Ph}$. (2012) parle de lecture silencieuse pour l'interprétation du texte. Comprendre un texte, constitue le même genre d'activité que de participer à une communication: décoder un discours pour l'interpréter. Ainsi, les emphases de la chaîne parlée, permettant d'articuler les différentes composantes d'un discours articulé, sont aussi présentes aussi bien en lecture silencieuse qu'en lecture à voix haute.

10 L'interprétation de l'oralité dans l'écriture se fait en partie par des contraintes graphiques (différenciation des homophones, ponctuation, interjections, signes d'oralité, etc.) surtout dans une langue en perte de morphologie orale comme le français. Le choix entre les diverses images acoustiques d'un même mot lexical (mot phonétique interprété par la syntaxe) dans le syntagme ${ }^{18}$ est possible par 
"l'orthographie». Cela est vrai aussi pour les différentes modalités (assertive, interrogative, exclamative ${ }^{19}$ ) dont les composantes des morphèmes oraux ne sont pas toujours évidentes.

11 Souvent, l'illusion d'un référent provoque de mauvaises interprétations phonologiques car on veut transcrire un objet linguistique qui n'existe pas. Telle est la puissance iconique de la mémoire de l'écriture. Cela se produit surtout en apprentissage d'une L2.

L'analyse du discours (même erratique) est bien différente d'une simple description grammaticale. Il s'agit d'aborder le discours par ce qui est impliqué dans l'acte de communication (locuteur et interlocuteur) pour identifier la syntaxe effective. Syntaxe et grammaire ne peuvent être confondues dans une approche phonologique de l'énonciation: la syntaxe organise les éléments d'une langue de quelque type que ce soit ; la grammaire étudie les structures figées d'une langue qui a été répertoriée.

13 Avec la syntaxe, on observe l'allitération de structures syllabiques souples qui peuvent conduire à l'écriture automatique (verlan, rap, joutes, jeu de mots,...): la langue «bourgeonne» de partout ${ }^{20}$.

Avec la grammaire, on aura des " faits de langue en boîte » : une langue en morceaux.

Cependant, ces deux approches sont complémentaires dans l'analyse discursive. Ainsi, dans les exemples qui suivent ${ }^{21}$, une seule approche ne peut pas rendre compte du sens :

1. Interprétation grammaticale :

*Qui l'eût grue $\square$ [le succès de l'utilisation de la grue géante au coût excessif]: le contexte définit la structure au niveau du syntagme (subjonctif plus-que-imparfait ou conditionnel passé $2^{\text {ème }}$ forme). C'est le participe passé qui permet le jeu syllabique /kby/vs/gby/, tout en transgressant dans le même acte la structure grammaticale.

2. Interprétation lexicale :

*La corde au fou $\square$ [suicide d'un détraqué en prison]: dans ce cas, ce n'est pas dans le syntagme que l'on interprète le sens voulu (référence à l'expression figée "la corde au cou"), mais dans la macro-syntaxe des éléments qui permet de mettre en rapport l'expression figée et une interprétation syllabique textuelle.

3. Interprétation syntaxique :

*Glandeur et décadence $\square$ [critiques du président de la commission européenne] : l'effet stylistique revient à la coordination d'une nominalisation atypique du verbe "glander" et d'un nom. Mais ici aussi c'est dans le mot phonétique que l'interprétation de la syllabe se fait par contraposition (grandeur $\square$ glandeur). Cela dépasse la simple explication grammaticale.

4. Interprétation sémantique :

*Un chômage très radieux $\square$ [préoccupation de l'expansion du chômage à tous les secteurs] : ni le syntagme, ni la macro-syntaxe ne peuvent expliquer l'impact de la formule. L'emploi atypique de "radieux" dans ce contexte renvoie à un paradigme sémantique.

Comme le montrent ces exemples, la syllabe se construit dans le discours qui l'informe, permettant les interprétations alternatives. Dans un discours, les segmentations (à tous les niveaux) dépendent de la compétence phonologique à dissocier l'image iconique de la syllabe acoustique.

\section{L'imaginaire dans le mot phonétique}

Considérons maintenant des exemples d'enseignes de magasins de Paris qui explicitent l'opérativité de l'image acoustique (renvoi à la culture commune, aux valeurs de la 
civilisation actuelle, aux signes linguistiques,...) dans la création du gabarit syllabique. Comme pour les exemples précédents, une analyse simple ne permet pas de rendre la force des réclames commerciales :

* Le chat beauté $\square$ [institut de beauté] évoque une image littéraire qui renvoie immédiatement à la parure. L'impact de la réclame est syntaxique car l'orthographe rend évident le glissement dans les positions syntagmatiques (botté : adjectif/beauté : substantif).

*L'ange Vins $\square$ [magasin de vins]. C'est une double image acoustique (région d'Anjou avec ses connotations traditionnelles) et la représentation d'un ange (à l'enseigne) qui interpelle le récepteur du signe.

${ }^{*}$ TrèsOr $\square$ [bijouterie]. L'or, bien capital ${ }^{22}$, est nuancé dans le mot «trésor » qui reçoit une image lexicale renouvelée par l'accent de la syllabe /tbe/ renvoyant à l'adverbe.

* La vie en robes $\square$ [boutique de vêtements] provoque une image acoustique mélodique ; l'icône comme pouvoir métaphorique exprimé par une "anaphore culturelle (la vie en rose) non explicitée.

19 Les interprétations de ces enseignes sont très complexes car la valeur iconique se fige au niveau de la syllabe que le récepteur/cible doit interpréter. Ces syllabes figées dans leur contexte sont donc des structures vivantes, comme les mots dans la langue.

Considérons du point de vue phonologique les exemples suivants :

${ }^{*}$ Miss Yoo $\square$ [Magasin de vêtements pour femmes]

*Class Croûte $\square$ [Brasserie élégante]

*Soft ki peut $\square$ [Magasin de compléments à Montmartre]

21 Ces enseignes dépassent les frontières des langues, laissant présumer que la phonétique est le soutien de la syntaxe par l'organisation d'éléments nucléaires qui s'articulent dans le message. Le signe linguistique est arbitraire, mais il est motivé par les figements langagiers que la communication verbale a produits en diachronie.

La première des enseignes, "Miss Yoo ", a été localisée dans un quartier populaire, mais on ne peut pas douter que la double interprétation syntaxique des mots soit accessible à tout un chacun (ou chacune) pour peu qu'on prenne le temps de la regarder. Elle est pourtant complexe et sollicite un bagage linguistique en anglais.

L'impact du message dans « Class Croûte » vient des registres convoqués : anglicisme à la mode et mot français (registres soutenu et populaire).

Dans « Soft ki peut ", les syllabes réfèrent à la fois à l'informatique et à la douceur, mais appartiennent aussi à une expression familière française ("sauve qui peut »). Elle cible un public de jeunes femmes avec une référence possible à l'internet (soft ware): libération, besoin d'évasion?

La productivité de ces enseignes vient de la complexité du mot phonétique qui oblige à inverser l'analyse linguistique pour aller du plus complexe au plus simple, de l'énoncé organisé à une syllabe aux positions souples qui s'adaptent à des contextes discursifs hétérogènes en rapport avec une macro-syntaxe.

L'image acoustique peut renvoyer à plusieurs langues et dépasse en cela l'interprétation grammaticale. Cette modification convoque de plus en plus le graphisme à travers l'image. Une nouvelle communication impose ses règles au moyen des codes multimodaux provoquant une «a-culturation " dont nous sommes victimes par le fait de notre rôle social de client dans le monde globalisé. 


\section{La syllabe iconique : binarité et diglossie}

Anscombre (2012) propose un double schéma interprétatif par troncation des constructions parémiques (proverbes). Ces expressions ne sont pas altérées par la variation diachronique et leur syntaxe est similaire à celle de la syllabe figée dans le texte, car leur définition suppose une "définition dénominative et [une] définition médiative ", un "ON-locuteur" (Anscombre, 2012) capable d'interpréter, dans l'axe paradigmatique, une expression qui perturbe l'axe syntagmatique de l'énoncé qui l'inclut.

Le patron fréquentiel des empans intonatifs qui composent les expressions figées suppose des "matrices rythmiques", binaires, qui font d'elles des clusters idiosyncrasiques dans les langues. Pour conserver leur "iconicité", ces expressions sont perçues comme décalées par rapport à la distribution métrique générale de l'énoncé ; elles signifient donc aussi parce qu'elles obligent à un «ajustement de débit tendant à égaliser les durées d'énonciation» (Martin, 2012). Comme structures formelles, elles peuvent être réutilisées par troncation pour obtenir une réaction active de l'interlocuteur. Si on utilise par exemple l'expression "quand on parle du loup... » [hablandodelrey de Roma...], tout le monde s'attend à voir arriver quelqu'un, même si le proverbe a été tronqué. L'intonation sera marquée par un trait de continuité (non descendant) au lieu du trait conclusif (descendant).

Pour d'autres proverbes, la troncation sert à nuancer un phénomène syntaxique :

- «tel père / tel fils» [de talpadre/ talhijo] : identification;

- «qui trop embrasse /peu étreint $»^{23}$ : contraste de positions syntagmatiques ;

- «à quelque chose malheur est bon»: contraste de signifiants (polarité des expressions: le malheur n'est bon / à rien).

La "parole exemplaire» se comporte dans le discours comme une para-syllabe (autosegment complexe) qui constitue une combinaison phonétique à laquelle on accorde des valeurs phonologiques propres. Entre le mot phonétique et le syntagme, l'énoncé parémique contribue à enrichir le sens de la phrase par les effets iconiques de sa syntaxe complexe dans l'imaginaire linguistique de l'ON-locuteur. Pour l'apprenant, elles sont un bon entraînement à la pratique de la langue car elles rendent évidente l'importance du schéma intonatif dont dépend la syllabation. Leur emploi est source de diglossies même chez les locuteurs très experts car leur structure syntaxique bouleverse l'énonciation et produit des effets intonatifs particuliers.

La conscience de l'autonomie des signifiants que nous révèlent les expressions figées libère l'apprenant de la hantise du signifié. Dans ces expressions, en effet, l'image acoustique et la macro-syntaxe ou syntaxe prosodique s'associent pour produire du sens. Déjà au niveau du mot phonétique, dans le cas de "Miss yoo " par exemple, la syntaxe de l'énoncé est indépendante des catégorisations grammaticales et exige une interprétation acoustique au niveau syllabique pour que le message rende toute sa valeur de simple réclame (Miss Yoo), d'interpellation (you, Miss !) ou même d'évocation (someone miss you), qui resterait opaque si l'interlocuteur visé ne collaborait pas.

La syllabe phonologique signifie par la syntaxe rythmée de ses éléments; le mot phonétique discrimine son interlocuteur qui doit sélectionner des signifiants ; les deux pôles de l'interprétation (signifiant et interlocuteur) conforment dans l'énonciation les 
mots prosodiques Martin (2009). En d'autres termes, l'émetteur et le récepteur contribuent à interpréter les éléments nucléaires d'un l'énoncé. Le signifiant n'est pas accessible autrement.

Une certaine diglossie semble être à l'origine de la codification linguistique : elle établit une différence entre le L-locuteur contraint par l'acte de parole et le ON-locuteur capable de l'interpréter, mais aussi de mal l'interpréter, ou de le modifier.

Revisiter la syllabation comme un ensemble de signes iconiques articulés, signifiant par la syntaxe de ses constituants nucléaires à la façon des expressions parémiques, dépasse l'analyse linguistique stricte. On pourra alors se servir méthodologiquement de ce caractère "diglossique » de l'interaction verbale pour considérer les L-productions des locuteurs inexperts comme base d'apprentissage d'un discours hésitant en construction. Des patrons interprétatifs peuvent alors être dégagés pour reconstruire la macro-syntaxe, ce qui ne peut pas être fait directement si l'on considère les énoncés un à un et donc déviants. Les énoncés conformes à l'intérieur du discours diglossique nous aideront ainsi à chercher les insuffisances perçues dans la construction textuelle pour y remédier.

Ainsi, la segmentation opérative construit aussi chez le locuteur inexpert des "énoncés stéréotypiques, qui fonctionnent comme un code référentiel commun au locuteur et à son destinataire ", en termes d'Anscombre (cité par Oddo, 2012 : 141).

\section{De syllabe iconique à la syllabation prosodique}

Dans le cas des mots d'esprit, la même stratégie de transgression du code scriptural, analysée dans les exemples supra, agit comme déclencheur de sens. Chez l'apprenant cette transgression est involontaire car il découvre petit à petit le sens des mots phonétiques par l'effet iconographique de la syllabe. Pour améliorer les compétences d'expression il faut travailler les mots phonétiques dans leur valeur iconique, les rendre signifiants et en faire de vrais morphèmes intonatifs ou des unités porteuses de sens (Chafe, $1994: 57$ ).

L'oral des apprenants comporte des collisions accentuelles, des pauses non conformes et différentes réalisations segmentales atypiques qui sont à la base de l'organisation syntaxique déviante de l'énoncé. Pourtant, ces mêmes occurrences phonologiques, loin de signifier une limitation expressive, assurent la communication chez le locuteur expert. Une diglossie originaire assouplit et rend possible l'interaction verbale. Faute de connaissances linguistiques, c'est la compétence phonologique qui assure la communication en accordant aux composantes de la chaîne parlée une valeur iconique par des proéminences syllabiques qui sont des indices pour l'interprétation du message.

Pour Pulgram (1979: 40) « the syllable exists, as it were, only for the sake of its boundaries », et contient «one and no more than one phonological vowel». Le repérage de cette voyelle phonologique dans le discours des apprenants semble une piste pour reconduire les productions erratiques dans le mot phonétique. En d'autres termes, les voyelles phonologiques dans la syllabation supposent un «ON-locuteur et un L-locuteur»: le ON-locuteur fait de l'apprenant un locuteur efficace, le L-locuteur produit un discours hésitant mais compréhensible par morceaux. Les décalages entre les productions du locuteur en difficulté et les énoncés normés signalent alors des positions phonologiques 
déviantes par rapport à un pic de sonorité (voyelle phonologique) car « if a languageis to funtioneffectively, a speaker isobliged to categorize a sharedreferent in a waythatallows the listener to identifyit " (Chafe, 1994 : 97).

Comme on le voit, le double point de vue (On-locuteur/L-locuteur) organise le message autour de positions phonologiques que l'on doit différencier alors d'autres positions non phonologique (extramétriques ${ }^{24}$ ) dans le comptage syllabique de l'énoncé. Nous avons convenu d'une dynamique qui explicite les rapports entre ces deux positions ailleurs $^{25}$ dans notre corpus de FLE. Cela implique de tenir compte de limites perceptives (chutes de sonorité) par rapport à l'image acoustique dans le mot iconique.

Différentes prosodies construisent différents mots phonétiques. La valeur phonologique que l'on accordera au mot phonétique, sa valeur iconique, doit être interprétée dans une dynamique à caractère métrique. Cette dynamique joue sur les positions syntaxiques par syncope perceptive ou transformation libre de segments non phonologiques. Ces phénomènes sont très présents en poésie ou chanson (phénomènes de rime, répétitions, oppositions).

41 Des contraintes rythmiques rendent donc possible l'articulation des segments pour faire de la syllabe une unité nucléaire souple servant, entre autre, à l'interfaçage entre les langues (intercompréhension) mais aussi entre les différents types ou genres de discours. Des patrons rythmiques communs et universels servent à transmettre l'information phonologique par le poids pondéré d'éléments segmentaux qui ont été décrits dans la morphologie de la syllabe (Klein, 1993) et décrivent la transmission de l'information dans le canal communicatif. En enseignement des langues étrangères, il est important de rendre visible à l'apprenant les contraintes prosodiques qui mettent en rapport les différents éléments du discours de la position segmentale première à la syllabe proéminente, de la syllabe proéminente au mot phonétique, du mot phonétique au mot prosodique qui se constitue en syntagme dans l'énoncé et fait partie d'un discours. Cet agencement successif d'éléments produit une logique phrastique dans le cadre macro-syntactique de la communication.

Les stratégies que l'on a analysées dans l'interprétation des expressions figées et de l'écriture inventive témoignent de cette composition syllabique à caractère intono-accentuel « en caché » dans la communication effective. La syllabation est une procédure dynamique complexe en ce qu'elle peut dépasser les limites imposées par la grammaire d'une langue (glissements syntaxiques, mots transparents, effets de style, jeux de mots,...) au service de la communication expressive. Plusieurs approches psycho-linguistiques comparent la compétence phonologique à la capacité d'emmagasiner et de coder les impressions de la vue. Ce rapprochement semble productif pour expliciter l'association entre image iconique et image acoustique dans l'acte de communication car "the ability to activateonly one new idea per focus of consciousnessseemsdepend on limitations inherent in human mental processing, regardless on the language one speaks » (Chafe, $1994: 159)$.

\section{Fréquence des patrons intono-accentuels dans la construction du syntagme}

Quand on analyse des corpus de locuteurs non experts, on peut remarquer que des patrons accentuels se reproduisent dans la syllabation en contact avec la langue cible. 
Souvent ces patrons accentuels provoquent des problèmes de phonotaxe ${ }^{26}$ et empêchent les syllabes proéminentes de se réaliser (gabarit non disponible dans l'inter-langue). Ce handicap excite des capacités phonologiques en activant des stratégies de mémoire motrice pour rendre plus facile la codification du message pour un destinataire. Le rôle de l'enseignant est de rendre consciente cette stratégie. Considérons alors ces exemples tirés d'un corpus d'apprenants de FLE ${ }^{27}$ :

1.... DE de la ville de Lyon

$<\mathrm{CH}$ : hum hum $>$

QUE J'ai/ auquel j'ai habiTE pendant/ un mois

ET J'ai des bons/ souvenirs

$<\mathrm{CH}$ : hum hum

DE cette expériENCEet de cette ville/ parce que j'étais très/z/heureux $1 / \ldots$

La double formulation des relatifs (que j'ai / auquel) est entraînée par la fréquence de la forme enregistrée dans l'inter-langue. L'apprenant hispanophone recourt souvent à la forme composée par ajout d'une position métrique qui interfère (préposition obligatoire devant le relatif en espagnol [en la que hevivido] mais qui n'est pas nécessaire en français.

Pour y remédier il faut rappeler la différence d'utilisation des deux formes possibles en français (avec et sans préposition).

2. $<$ CH : je euh j'ai visiTEj'ai euh j'ai FAIT une courte séANCEà LYON

[continue sans pause]>

Le mot déviant (séance) est plus immédiat chez cette informatrice qui cherche un mot féminin (" estancia » en espagnol). Le mot «séjour » est pourtant bien connu par les locuteurs de ce niveau. Il est aussi possible que l'ajout de "coda» au masculin («court » prononcé /kurt/) soit une difficulté pour l'anticipation du mot phonétique dans le syntagme. Quelle que soit l'interprétation, il faut travailler sur les possibilités lexicales par rapport à l'axe paradigmatique déterminé par le syntagme pour remédier au problème.

3. Le LA Rhône et la Saône la SaONE c'est / le !euh/ LE fleuve / qui

$<\mathrm{CH}$ : hum hum>

Qui *embouche* dans la Rhône

Le vocabulaire spécifique fait défaut (« embouche» vs "se jette»). La construction syntaxique du verbe "emboucher» se fait naturellement à partir du mot lexical « embouchure ». Cette formation n'est pas répertoriée dans la langue figée ${ }^{28}$ et est, en conséquence, déviante. Ces figements de mots en diachronie, probablement arbitraires, ne peuvent que poser problème aux locuteurs inexperts.

La compétence à utiliser la stratégie syntaxique pour construire l'énoncé doit être mise à l'avantage en rappelant, par exemple, des occurrences où la dérivation est possible (nominalisation, adjectivation,...).

4. Et / bon / euh / surtout je me souviens de l'eau /de / de la clarit-de le euh mh

*)*/ de l'eau verte / presque verte/ qu'il / QU'IL emporte

Des difficultés se produisent au niveau de la syllabation : «clarit». La locutrice est sensible à l'interférence du mot " claridad » [transparence] de l'espagnol et se corrige. Cette stratégie de correction est une stratégie-clé dans l'apprentissage car elle génère un mécanisme de compensation (mot lexical alternatif « verte ») et une répétition qui facilite la transmission de l'information. L'accent distinctif sur la deuxième occurrence de «verte» renvoie immédiatement à la qualité de l'eau. L'enseignant peut alors 
rappeler comment décrire l'eau en paraphrasant (je me souviens de l'eau claire, transparente,...).

5. <CH ; on *peuvait* on pouVAITèh *NA- *navirer* naviguer scette dérivation de navire produit-elle une consonne alternative $(/ \mathrm{r} / \mathrm{vs} / \mathrm{g} /)$ ? Les productions syntaxiques alternatives des locuteurs non experts, pourraient-elles nous faire découvrir des règles de phonotaxe dans l'évolution des langues en diachronie? dérivés du verbe (navigation, navigable,...) et des synonymes de "navire " (bateau, barque,...).

6. Ce sont des villages / comme euh de l'âge euh / médiéval / médiév- de le médio euh *medioevo*/ médiéval/

$<\mathrm{CH}$ : médiéval oui

Je pense que ça se dit comme euh * médieval ${ }^{*}$ / et tout /t/ en en pierre dorée

Cet exemple (6) est conclusif : il présente l'autonomie du signifiant par rapport au signifié en FLE, mais aussi en phonologie. La locutrice, dont la prononciation est excellente, n'arrive pas à reconnaître l'image acoustique du mot français «médiéval ", car sa prononciation est très proche de l'espagnol «medieval ». Cette coïncidence lui suggère le pluriel qui s'ajuste plus à une différence phonétique recherchée (villages médiévaux). Or, elle a bien dit «... villages de l'âge médiéval », où le pluriel n'est pas pertinent. Un écho de /o/ phonologique provoque l'hésitation qui suggère « medioevo » (moyen âge en espagnol) pour « médiévaux ».

Ces appréciations restent des conjectures ouvertes à des recherches futures. Or, tout probablement l'icône d'une image acoustique non disponible en français a produit la diffluence dans la logique de la construction syntaxique du discours. Ces exemples relevés dans les corpus oraux confirment la procédure très complexe de la verbalisation en langue étrangère qui reste dépendante de la mémoire phonétique. L'icône produit par la mémoire acoustique semble une bonne base pour l'apprentissage.

On peut conclure alors que l'énonciation en apprentissage est déterminée par :

- la limite imposée par les structures apprises (en grammatisation) inventoriables ou non (exemple 1);

- la fréquence de la prononciation des mots appris pouvant provoquer des blocages phonotactiques et/ou des diffluences (exemple 2);

- la mise en œuvre de stratégies syntaxiques de l'inter-langue pour ajuster les axes paradigmatique/syntagmatique et produire une énonciation effective (exemple 3) ;

- l'anticipation des mots phonétiques à caractère iconique (exemple 4);

- le repérage des positions segmentales nécessaires à la verbalisation (5);

- la priorisation du signifiant sur le signifié (6).

\section{La fonction de la mémoire dans la transmission du sens}

Les exemples ci-dessus montrent comment opère l'inter-langue dans l'énonciation chez le locuteur inexpert. La transmission du message est fragile et s'appuie sur les fonctions émotive et phatique de la transmission du message. Chez le locuteur expert, ces fonctions ne sont pas particulièrement privilégiées car le bagage de l'inter-langue 
(lexical, phono-tactique) est beaucoup plus important, ce qui lui permet d'avoir une expression plus souple et plus aisée sans recourir à la situation extra-linguistique. Quand on s'exprime en langue maternelle, il existe peu de contraintes invoquées par le besoin pragmatique de la transmission de l'information. Notre discours est significatif (grammatical) par définition, on peut alors s'efforcer de cibler le message lui-même pour le rendre plus effectif. Les stratégies langagières ( 3 - 6 supra), utilisées inconsciemment par le locuteur inexpert, sont mises à l'avantage, chez le locuteur expert, pour être utilisées dans un but expressif et pour rendre plus effectives les icônes d'un bagage interculturel linguistique commun.

Nous pouvons donc conclure que, tant en FLE qu'en analyse du discours,

- les compétences qui mettent en jeu la syntaxe active inter-langues servent à nuancer l'énonciation comme on l'a vu dans les exemples supra (presse, enseignes);

- les compétences qui anticipent des mots phonétiques alternatifs sont au service de l'expressivité (enseignes de Paris);

- les compétences qui se servent de la syllabe phonologique dans le mot phonétique permettent de jouer sur les signifiants (fonction poétique);

- les compétences privilégient le signifiant sur le signifié pour dépasser les contraintes imposées par les grammaires des langues (mots et expressions transparents) dans l'élaboration du discours.

Dans une approche phonologique de l'énonciation, les genres discursifs embrassent la totalité du dire tant chez le locuteur expert que chez le locuteur inexpert, et le signifié émerge des rapports syntaxiques entre des signifiants-indices que l'on répertorie dans la mémoire phonologique par groupes syntaxiques. Ceux-ci reproduisent les empans intonatifs du discours. En effet, « anyonewholistensobjectively to speech willquickly notice thatitis not produced in a continuous, uninterrupted flow but in spurts » (Chafe, 1994: 57). On voit alors dans quel sens le signifié est un objet de communication inanalysable si ce n'est par référence aux icônes qu'il produit par l'image acoustique de ses composantes dans le syntagme.

L'accès à l'analyse d'un discours (imparfait ou non) se fait par les figements syntaxiques relevés dans l'énonciation. Comme on le vérifie dans les discours d'apprenants, il s'agit de procéder, dans la verbalisation, à des segmentations qui répondent à des effets intono-accentuels que l'on peut mesurer dans le rapport entre perceptibilité et non perceptibilité de position métriques. Qu'indiquent donc les tronçons, épurés, d'énoncés conformes ou non dans le discours atypique des apprenants?

Nous avons fait un décompte des soudages syntaxiques, aidés par les contextes de liaison, car celle-ci semble avoir une fonction "intégrative" (Côté, 2012) ${ }^{29}$. Sur 250 occurrences relevées, le résultat indique que des catégories syntaxiques peuvent émerger dans les empans intonatifs perçus :

1. Présentatifs («ce + verbe être »): 59 (soudages 30, liaisons non réalisées: 29): cette catégorie concerne les formules impersonnelles du type "ce+verbe être». Elles sont fréquentes et indiquent l'absence de syntagmes verbaux à sujet personnel; l'équilibre presque à $50 \%$ entre liaison réalisées et non réalisées permet d'affirmer que le discours reste pauvre (pas de sujets personnels et nominalisations vagues: c'est une chose/ personne). L'apprenant recourt à des rapports anaphoriques et à des inflexions intonoaccentuelles pour permettre que l'interprétation des signifiants aboutisse.

2. Soudage nominal (déterminant + nom) : 35 (non réalisées : 2) : le haut pourcentage de réalisation (presque à $95 \%$ ) indique que la structure syntaxique est acquise, mais seuls les 
déterminants monosyllabiques sont privilégiés : la liaison obligatoire est reconnue, mais la liaison variable est peu présente. Il faut alors travailler la diversité des mots déterminants en incluant des polysyllabiques ${ }^{30}$. Une approche par les genres de discours serait nécessaire pour enrichir le lexique et les structures syntaxiques à ce niveau (textes de type économique, social, journalistique où des spécificateurs de nombre sont de rigueur : divers, plusieurs, différents, quelques,...).

3. Soudage verbal (verbe + sujet + complément d'objet) $: 45$ (non réalisées ${ }^{31}: 14$ ) : ce groupe peut se présenter comme explication de l'emploi excessif des formes présentatives constatées (voir supra) : mêmes réalisations au total (59) que pour celles-ci, mais moins de soudages vérifiés. Le locuteur n'est pas sûr de la morphologie qui ne correspond pas à son expérience inter-linguistique (absence de morphologie orale, patrons métriques décalés dans l'emploi des désinences). Les groupements syntaxiques restent peu fréquents et produisent des diffluences ; les textes narratifs sont alors à privilégier.

4. Modificateurs verbaux - Circonstants / mots de rapport: 87 (non réalisées : 42) : cette catégorie indique que les adverbes, ainsi que d'autres mots de liaison (liens logiques), lient relativement peu. Ces mots lexicaux doivent donc être travaillés à l'intérieur des discours argumentatifs pour bien marquer les nuances de la variation possible dans les modalités (mai/z/il va venir... / mais/?/il ne viendra pas !) ${ }^{32}$.

60 Pour que le message puisse donc être mieux transmis, à l'oral, des positions segmentales doivent être interprétées (consonnes de liaison, enchaînements, syncope de positions vocaliques). Apprendre que les positions segmentales sont souples dans le syntagme en français aide le locuteur non expert à transmettre l'information en adaptant les patrons métriques de son inter-langue aux types de discours. De subtiles différences de segmentation aident à acquérir de nouveaux patrons intonatifs nécessaires à la construction textuelle dans le cadre de la macro-syntaxe: phrases conclusives (il nous l'a appris quand/t/il est/t/arrivé $\square$ ) / non conclusives (quand/t/il est/ $\mathrm{P} /$ arrivé... $\square \square$ on/n/a mangé). En outre, la segmentation peut justifier les choix syntaxiques d'une grammaire de langue ${ }^{33}$ comme dans ces exemples d'inversion interrogative - prendra-t-il le métro?, vas-y, et ne voilà-t-il pas qu'il m'engueule!révélateurs d'une complexité grammaticale d'ordre phonologique.

61 L'analyse des contextes de liaison montrent aussi qu'il faut veiller aux enchaînements parasites. Pour l'enseignement du FLE, l'approche discursive indique que le phénomène $\mathrm{du}$ non enchaînement intervient dans la segmentation autant que la liaison pour produire du sens. Centrer l'apprentissage de la syntaxe en partant de l'énonciation effective semble raisonnable et constitue le principe de base de l'approche phonologique que nous proposons, les mots phonétiques fixant, par segmentation, des icônes responsables de la transmission de l'information dans la syllabation. Ces icônes produisent des inflexions dans le débit articulatoire à caractère intono-accentuel dont on ne peut percevoir, en tant qu'On-locuteur, que les ruptures. La chute dans la perception de sonorité délimite alors le mot phonétique dans le syntagme. Cela est avéré tant chez les locuteurs experts que chez les inexperts qui s'appliquent à construire leur discours.

Une écologie de la langue dépendante de normes discursives aide l'apprenant à contrôler un français épuré, où l'intonation a du mal à s'installer. Pour maîtriser les genres discursifs et construire du sens, une étude des contextes de liaison s'avère nécessaire. La substance discursive dépend d'une syntaxe construite avec un matériel phonologique responsable de la transmission de l'information. L'impact des formules iconographiques (titres de presse, publicités, expressions figées, poésie, jeux de mots,...) 
témoignent de cette particularité phonologique de la communication. Il n'y a donc que les étrangers s'évertuant à vouloir maîtriser une langue non maternelle qui produisent "une langue neutre, standard".

Le domaine de l'apprentissage des langues étrangères est l'analyse du discours actif où se construisent les faits de langue chez les locuteurs natifs. La formation de groupes de sens est donc préalable à l'acquisition de la prosodie, et par là, à la reconnaissance de la macro-syntaxe.

Cela rapproche l'apprentissage des langues étrangères de l'analyse de la linguistique textuelle et invite à une méthodologie où des modèles métriques sans constituants (Laks, 1997) opèrent dans la construction du discours, par la reconnaissance de la valeur iconique des productions syllabiques. La syllabe a une existence virtuelle que l'interlocuteur peut seulement interpréter dans la verbalisation.

Les expressions phrastiques qui se caractérisent par leurs contraintes rythmiques présentent bien la différence entre un mot phonétique et un mot prosodique. La prise de conscience du modèle métrique impliqué dans la syllabation sert à établir le rapport entre prosodie et syntaxe. Être conscient de ce lien formel et le privilégier rendent la communication en milieu plurilingue plus efficace et invite à l'analyse discursive d'un point de vue phonologique.

\section{BIBLIOGRAPHIE}

ANSCOMBRE, J-C., «Pour une théorie linguistique du phénomène parémique », in ANSCOMBRE, DARBORD, ODDO (dirs), La parole exemplaire. Introduction à une étude linguistique des proverbes, Paris, Armand Colin, 2012, pp. 21-39.

CHAFE, W, Discourse, consciousness and time. Chicago : Chicago University Press, 1994.

CÔTÉ, M-H., « Des configurations aux constructions : retour sur la liaison et ses déterminants », 10

èmes rencontres Colloque RFP 2012, Actes des Communications Orales, 2012.

ENCREVÉ, P., La liaison avec et sans enchaînement. Phonologie tridimensionnelle et usages du français. Paris, Seuil, 1988.

FERNÁNDEZ-ECHEVARRÍA, M-L., Contextes de liaison et FLE. Productivité des positions / / /, /t/, /n/ et /z/, Thèse de doctorat, Université Paris X-Nanterre, 2013.

GADET, F. \& TYNE, H., « La séduction du binaire », in POOLEY\&LAGORGETTE (eds), On linguistic change in French, Chambéry, Université de Savoie, 2011, pp. 55-67.

HARRIS, J.W., La estructura silábica y el acento en español. Madrid, Visor, 1983-1991.

KLEIN, M. « La syllabe comme interface de la production et de la réception », In LAKS\&PLENAT, (dirs.), DE NATURASONORUM. Essais de phonologie, Saint Denis : Presses Universitaires de Vincennes, 1993 pp. 100-134.

LAKS, B., Phonologie accentuelle : métrique, autosegmentalité et constituance. Paris : CNRS, 1997. 
LAKS, B., « La liaison et l'illusion », Langages n 158, Larousse, 2005, pp. 101-126.

MARTIN, PH., Intonation du français, Paris, Armand Colin, 2009.

MARTIN, PH., «Intonation, rythme, eurythmie de locutions et proverbes français ", in ANSCOMBRE, DARBORD, ODDO, (dirs), La parole exemplaire. Introduction à une étude linguistique des proverbes, Paris, Armand Colin, 2012, pp. 159-169.

ODDO, A., « Phénomènes de troncature », in ANSCOMBRE, DARBORD, ODDO, (dirs), La parole exemplaire, Introduction à une étude linguistique des proverbes, Paris : Armand Colin, 2012, pp. 133-146.

PETIT, G., in (ed.) À la croisée des mots. Hommages à Taïeb Baccouche, Salah Mejri, Université Paris X, 2011, pp. 237-278.

PUlgram, E., Syllabe, Word, Nexus, Cursus, Mouton, 1970.

ROSSI, M., « Le Français, langue sans accent ? », in FONAGY \& LÉON (eds), L'accent en Français contemporain, Ottawa, Didier, 1979, pp. 13-51.

\section{NOTES}

18. Voir les différences entre eau, haut, « ô, au, oh !... »

19. Viens ! Vient, parlez ! Parler, parlait, parlaient.

20. Référence aux propos d'Encrevé dans une émission télévisée sur la langue française (TV5), en 2013.

21. Canard Enchaîné, $n^{\circ} 4836$, du 3/07/2013. Le sens se fige dans la déviance grammaticale par référence au contexte dans le domaine de la macro-syntaxe.

22. Rappelons les termes "Or noir", "Or liquide", et le grand nombre d'expressions métaphoriques dérivées.

23. Voir l'ouvrage de référence (Anscombre, Darbord \& Oddo, 2012) pour les explications exhaustives et édifiantes.

24. Voir Harris (1991) pour une étude plus précise de l'accentuation de l'espagnol.

25. Fernández-Echevarría (2013).

26. Phonotaxe : caractéristiques qui rendent possible la verbalisation de phonèmes (de phono et tactique).

27. Les extraits sont puisés dans mon corpus d'apprenants de FLE analysé dans ma thèse de doctorat. Les transcriptions respectent un code d'annotation qui apparaît dans les textes présentés et que je ne modifie pas pour plus de transparence.

28. «Emboucher" pourrait avoir comme seul champ lexical possible «bouche», et non pas «fleuve » comme « embouchure».

29. Nous considérons comme des soudages syntaxiques les groupements syllabiques dans de possibles contextes de liaison (réalisée ou non) où ni les hésitations, ni les pauses, ni les chevauchements, ni les effets accentuels sur les mots lexicaux n'interrompent le débit énonciatif. 30. Seuls deux déterminants polysyllabiques ont été relevés.

31. Les occurrences concernent 2 polysyllabes : différents acteurs, plusieurs expéditions.

32. Pas/z/un seul, pas/z/encore, pas/z/oublié, pas/z/intéressant... ce type de soudure aide à produire le syntagme conformément à l'empan intonatif marqué par la syntaxe dans le groupe de souffle. 33. Le signifiant actif peut même développer l'ajout de consonnes épenthétiques au niveau de la grammaire. 


\section{RÉSUMÉS}

Comment des énoncés déviants peuvent-ils produire un discours signifiant? Un corpus d'apprenants répond à la question par le dépistage d'un gabarit syllabique à caractère métrique qui opère efficacement dans l'acte de communication, malgré l'énonciation atypique, les répétitions et les formulations alternatives. Des exemples de la publicité, de la presse, de la parémiologie témoignent de cette stratégie langagière : une syntaxe à caractère binaire informe la syllabation pour justifier le sens véhiculé par le discours. Les syllabes deviennent des figements à caractère iconique servant à interpréter les différents genres d'énoncés, dans une approche phonologique de l'analyse du discours.

How can deviant utterances produce a significant speech? A learners' corpus answers the question. Indeed, some metrical syllabic distribution seems to operate effectively in communication act, in spite of the atypical syntactic constructions, multiple options utterances and observed repetitions. Various advertising, press and paremiology examples reflect this linguistic strategy: a binary syntax informs syllabification to justify the meaning conveyed by the discourse. Syllabic templates acquire then iconic typology allowing the interpretation of different speech utterances and making possible the phonological approach to discourse analyse.

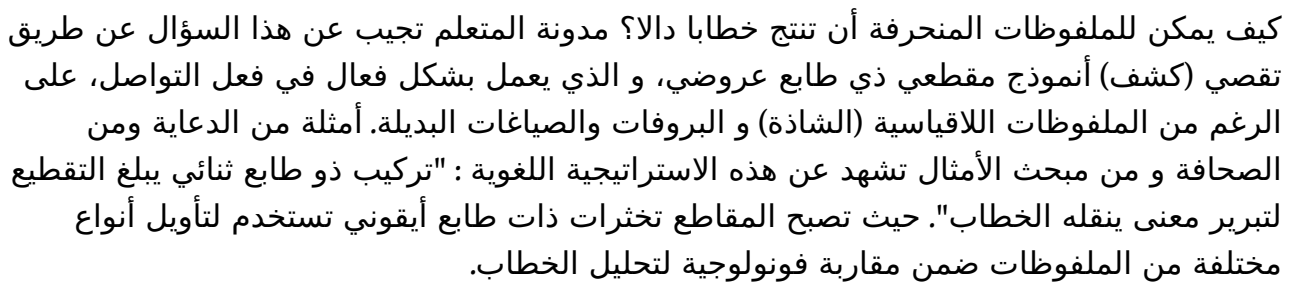

\section{INDEX}

Mots-clés : syllabe, iconicité, discours, parémiologie, métrique

Keywords : syllable, iconicity, discourse analysis, paremiology, metricity

مقطع لفظي, أيقونية, خطاب مبحث الأمثال (دراسة الأمثال), عروضفهرس الكلمات المفتاحية:

\section{AUTEUR}

\section{MARIA-LUISA FERNANDEZ-ECHEVARRIA}

Université de Complutense - Madrid - Espagne

Université Paris X - Nanterre - France 